\title{
Analysis on the Translation Errors of Chinese-specific Words in Government Work Report
}

\author{
Li ZHANG \& Xiaoyue WANG \\ School of Foreign Languages, North China Electric Power University, Baoding 071003, Hebei
}

\begin{abstract}
As a representative document of the government, Government Work Report consists of looking back at both achievements and defects of the past five years, which recently becomes the window for foreigners knowing about China. However, the most conspicuous feature of Government Work Report is the applications of Chinese-specific words and their translation errors, which causes cultural conflicts and misunderstanding easily. Based on the previous study of Chinese-specific Words in Government Work Report and 2007 to 2015 Government Work Report, this thesis addresses the translation errors such as mechanical counterpart, incorrect collocation, and misuse of commendatory and derogatory terms and the translation strategies in detail such as literal translation, free translation, literal translation plus annotation, transliteration. We attempt to apply them to the translation in order to convey the metaphorical meaning of Chinese-specific words, retain its national coloring and transmit Chinese culture.
\end{abstract}

KEYWORD: Chinese-specific words; Government Work Report; translation errors translation methods

\section{INTRODUCTION}

As a representative document of the government, GWR (Government Work Report) consists of all issues of general concern, covering all walks of life, from economy, politics, culture, education, foreign affairs, environmental protection, ethnic groups to social security, with emphasis varying with the priority of government work. The Government work Report belongs to a kind of political comment style, and the translation of Government work Report is quite important. However, many Chinese-specific words in Government work Report are full of strong national coloring, and have rich content, which makes them a hard nut to crack in translation.

\subsection{The definition of Chinese-specific words}

As for the definition of Chinese-specific words, they are defined as words which only exists in Chinese culture and can reflect the characteristic of China's history, culture, politic and economy such as "one country, two systems" .Chinese-specific words also reflect things, events, objects or concepts which are peculiar or unique to the Chinese cultures. such as "people's Commune", and "cultural revolution". Nearly all of them have rich cultural connotations, such as "golden mean".

\subsection{Literature review}

There are many articles to talk about the translation of Chinese-specific words. Error Analysis of Chinglish Vocabulary (Li Ying \& Hui changzheng, 2008) addresses the errors in the translation of Government Work Report and their reasons. Jia Yuling, one of the earliest pioneers who try to do some research on Government Work Report analyzes the reasons why Chinglish occurs and gives two ways to solve them in his article Study on How to Overcome the Tendency of Chinglish from the Translation Errors of Chinese-specific Words in Government Work Report.(Jia Yuling, 2003) Wei, Aiyun.in her article Attitudinal study on Chinese English(Wei, Aiyun, 2002) summarize some errors and problems of Chinese-specific words and tries to find methods to solve them.

We investigate the errors of Chinese-specific words in the English version of the Government Work Report and attempt to correct them and to apply new methods to English translation of Chinese-specific words to overcome difficulties faced by translators. 
2 TRANSLATION ERRORS OF CHINESESPECIFIC WORDS IN GOVERNMENT WORK REPORT

When speaking English, the habitual practices become a barrier for Chinese to think in the English way, which unavoidably produces some problems such as mechanical counterpart, Incorrect Collocation and misuse of commendatory and derogatory terms.

\subsection{Problems of mechanical counterpart,}

Mechanical counterpart means that certain Chinese expression is regarded as certain English items by English learners, which makes the translation inaccuracy. It often occurs when English learners do not understand the Chinese-specific Words thoroughly. There are such expressions:

(1) Issues of common concern (2010GWR)

(2)To be concerned about the living of the people (2010GWR)

Some English learners translate it literally and mechanically, which is really not concise, even incorrect. They just use the familiar expression unconsciously instead of understanding the Chinesespecific Words of "concern" and "living". In 2011, the first expression is translated as "issues of mutual interest" and the second one is translated as "to care about the well-being of the people". Translation itself is a kind of cognitive activity. Since all the people are in different circumstances, their angles of knowing the world are different. We should reorganize the sentence based on English habit from the point of English readers.

\subsection{Problems of Incorrect Collocation}

Word collocation refers to the language phenomenon that some words appear together with other words. When translating the source language into the target language, especially some Four-character words with deep meaning, incorrect collocation often occurs at that time. For example, "strong policy" (2009GWR) is an incorrect collocation. If the objects or materials are modified by "strong", they are not easily broken and can resist a lot of strain. So, to describe the "policy" of a country, "rough" is better. Another example, "realize self-sufficiency" (2009GWR). While "realize" should not collocate with the word "self-sufficiency" and "reach" is more appropriate.

To avoid the problem of incorrect collocation, it is necessary for English learners to thoroughly understand the Chinese meaning, and break away from the literal Chinese-specific words and phrases. Translators should adopt the appropriate collocations based on distinct cognition.

\subsection{Misuse of commendatory and derogatory terms}

Some English learners cannot understand the Chinese meaning thoroughly, so it's difficult for them to tell commendatory and derogatory terms, which produces the misunderstanding for foreigner readers. For example, "statesman" could not been translated as "politician", because "politician" is derogatory and negative in Chinese. Another example,

We must carry on active propaganda for environment protection. (2008GWR)

This translation is the typical one of misuse of commendatory and derogatory terms, because "propaganda" is negative and derogatory. So the corrected one "We must carry on active promotion to environment protection." breaks away Chinese habitual thinking.

\section{TRANSLATION METHODS OF CHINESE- SPECIFIC WORDS IN GOVERNMENT WORK REPORT}

Translation of Chinese-specific words requires not only to convey the meaning of the original as much as possible, but also to win the acceptance of the target readers. In this section some translation techniques are illustrated in detail in order to overcome the errors talked about above.

\subsection{Literal Translation}

Literal translation, which preserves the original image and form without causing confusion in meaning, aims to preserve the national flavor of the source language items, instead of going against the linguistic principles of the target language. Translators should make every effort to retain figurative images, rhetorical devices and national characteristics of the original languages as much as possible when the meaning of the words can produce the same effect as the original languages do. The following are several examples:

(1) Socialism with Chinese characteristics (GWR 2010)

(2) We deepen overall rural reform (GWR 2007)

The literal translations of Chinese-specific words not only keep the original form of the items, but also transfer the original image and metaphorical meaning effectively. Furthermore, it keeps the full flavor of the items and the highest degree of faithfulness. It also produces problems of mechanical counterpart and misuse of commendatory and derogatory terms and we translators should pay more attention to it. 


\subsection{Free Translation}

As Nida pointed that sometimes the translated text would be obscure or wrong if we stick to form equivalence by retaining the original images. (Eugene A. Nida, 2002) In free translation we should give priority to meaning rather than form. Free translation may be defined as a supplementary means to mainly convey the meaning and spirit of the original without trying to replace its sentence patterns or figure of speech, which deal with the problem of incorrect collocation efficiently. Examples are listed to illustrate this method:

(1)Development is the essential principle (GWR 2009)

(2)We took a holistic and balanced approach in employing fiscal policy. (GWR 2010)

In second example, free translation make the whole structure more concise with the collocation of "took a holistic and balanced approach". If the images of the source text are not familiar to the target readers, translators should change the images into the one that is more familiar to the target readers to avoid the odd collocation. Although the original images, metaphor and flavor are not kept, the meaning is successfully translated and meaning of Chinese-specific words has been successfully conveyed.

\subsection{Literal Translation plus Annotation}

To avoid the problems of mechanical counterpart, we translators could annotate the historical persons and events. Literal translation plus annotation is an efficient way to keep the external form and its cultural and metaphoric meaning, especially the word with number and the four-character words. Many Chinese-specific words contain allusions, legendary persons or events in Chinese culture.. By doing so, the formal equivalence and the functional equivalence can be achieved. To illustrate this method, the following is the specific example.

We should continue to increase spending on agriculture, rural areas and farmers; improve infrastructure and basic public service in rural areas; and promote integration of urban and rural development. (2013 GWR)

As the Chinese words abbreviates three aspects of agriculture, rural areas and farmers and the three aspects emphasize different things. If it is translated "three agricultures", the deep meaning apparently could not be understood by foreigners. So it is translated as "agriculture, rural areas and farmers" in GWR.

Another example:

"Five forms of support (food, clothing, medical care, housing and burial expenses)" (2012 GWR)

This is the manifestation that the government cares about people in five aspects. Even targat readers do not understand what five forms of support are, they can know the meaning clearly and quickly by referring to the annotation.

Literal translation plus annotation, to a certain degree, is literal translation. It conveys the meaning of the words correctly and keeps the original images which do not exist in the target language but needed to be known by the English readers for functional equivalence.

\subsection{Transliteration}

Some words are difficult to understand for the target language readers and easily produce the problem of mechanical counterpart, incorrect collocation and misuse of commendatory and derogatory terms if they are translated only by methods above. Transliteration, referring to the verbatim spoken representation of one language by another, would be the best way to deal words of such kind according to their pronunciation. With the uprising of China, more and more Chinese-specific are accepted by the English-speaking people. Actually, there are two methods of transliteration: the first one is to transcribe Chinese-specific words directly in pinyin, such as yuan, jiao, fen; the second one is to imitate and approximate their pronunciations by spelling them in English words, such as kungfu, mahjong, wonton, Hong Kong, Taipei.

After introducing four translating techniques, here arose the problems. How to choose among these methods? Here is the principle. Literal translation is the most important method, by which the national color, image and metaphor of Chinese-specific words can be kept in its English translation. If the form and the meaning can not be kept at the same time, free translation is a better way to convey the meaning without keeping its form. If the allusions in are familiar to the target language readers, annotation can help the foreign readers understand a culturally loaded words. If the three methods can not do, transliteration is the best choice.

\section{CONCLUSION}

Chinese-specific words are heavily culturally loaded. Most of them have double sense: figurative sense and literal sense, which makes their translation a complex and difficult task. This thesis introduces some translation errors and analyzes four translation strategies for Chinese-specific words aiming to achieve functional equivalence in Chinese-specific words translation by analyzing a lot of examples.

First, the thesis lists some problems during the translation, including the mechanical counterpart, incorrect collocation, misuse of commendatory and derogatory terms. These problems can remind us that translators should master both Chinese and English well to avoid the same kind of mistakes. 
Second, this paper summarizes four strategies of translating Chinese-specific words. They are literal translation, free translation, literal translation plus annotation, transliteration, of which the first one is the most important, bearing the original national colors, images and rhetorical devices of Chinesespecific words. The author also gives a lot of examples to prove relevant arguments and explain when and why, use which translation method to achieve functional equivalence. Due to the differences between the two languages, it is impossible to achieve complete equivalence when Chinese-specific words are translated into English.

Besides, the thesis is limited in terms of depth and breadth owing to the limitations of the author's personal understanding and knowledge. The attempt here is to share an opinion in this field.

\section{REFERENCES}

[1] Eugene A. Nida. 1969. Toward a Science of Translating. Guangxi Normal University.

[2] Wei, Aiyun. 2002. Attitudinal study on Chinese English Master Degree Thesis. Guangxi Normal University.

[3] The Central People's Government of the People's Republic of China Website, [G/OL] http://english.gov.cn/official/workreport.htm. 2007-03-01/2015-03-06.

[4] JiaYuling, 2003, Study on How to Overcome the Tendency of Chinglish from the Translation Errors of Chinese-specific Words in Government Work Report, Shanghai Journal of Translators for Science and Technology(4):26-28.

[5] Li Ying \& Hui Changzheng, 2008, Error Analysis of Chinglish Vocabulary, Journal of XinYang Agricultural College (2):97-99. 